\title{
Coulisses
}

Revue de théâtre

19 | Hiver 1999

Varia

\section{L'art vivant, une utopie vitale en Franche-Comté}

\section{Robert Damien}

\section{CpenEdition}

Journals

Édition électronique

URL : https://journals.openedition.org/coulisses/5509

DOI : 10.4000/coulisses.5509

ISSN : 2546-9460

\section{Éditeur}

Presses universitaires de Franche-Comté

\section{Édition imprimée}

Date de publication : 1 janvier 1999

Pagination : 25-28

ISBN : 2-913322-09-3

ISSN : $1150-594 \mathrm{X}$

\section{Référence électronique}

Robert Damien, «L'art vivant, une utopie vitale en Franche-Comté », Coulisses [En ligne], 19| Hiver 1999, mis en ligne le 18 octobre 2019, consulté le 09 janvier 2022. URL : http://

journals.openedition.org/coulisses/5509; DOI : https://doi.org/10.4000/coulisses.5509

Ce document a été généré automatiquement le 9 janvier 2022

Coulisses 


\title{
L'art vivant, une utopie vitale en Franche-Comté
}

\author{
Robert Damien
}

Intervention de clôture de la journée de rencontres et d'échanges Scènes et spectacles vivants en Franche-Comté, organisée par le Conseil Economique et Social de Franche-Comté, le 7 avril 1998. Coulisses remercie le Président Jean-François Robert d'avoir bien voulu autoriser la reproduction de ce texte.

1 Je voudrais tout d'abord remercier le président Robert de m'avoir invité. Je ne vous infligerai pas un pensum, ni à titre d'expert ni à titre d'artiste, ce que je ne suis pas. J'essaierai simplement de réfléchir avec vous sur l'intitulé même de vos journées : scènes et spectacles vivants. La question est en effet celle-ci : comment donner vie, comment rendre vivant, comment faire vivre des scènes et des spectacles, comment les rendre en fait source et ressource de vie?

2 Votre insistance sur ce terme "vivant» m'a semblé curieuse, car c'est un terme biologique. Sans doute était-ce là un moyen d'éviter les termes plus polémiques, comme « contemporain », « actuel », « moderne »... En tous cas, votre volonté témoigne d'un véritable souci exigeant, ambitieux, si ce n'est même pour certains déplacé, voire utopiste. On en appelle en effet d'autant plus à la vie, qu'on sent bien qu'il existe un risque de mort... Revendiquer en effet la vitalité, la vivacité, c'est affronter une crainte de mort.

3 Quelle serait donc cette mort concernant les scènes et spectacles ? Cette mort-là serait désignée comme une inertie, une sclérose, une normalisation. Une normalisation dans quoi ? Il me semble que la lamentation actuelle à laquelle participent de nombreux collègues philosophes, sur la crise de l'art contemporain et de l'art moderne, nous signale que pour certains, le coma de l'art est déjà dépassé. Le mort est en fait embaumé. La chronique d'une mort annoncée indique déjà que les héritiers se disputent l'héritage... Et se partagent les dépouilles.

4 En effet, nous avons connu, depuis une vingtaine d'années, une sorte de fureur, mais cette fois pas de vivre... Excusez le caractère polémique de cette intervention, mais il s'agit de la fureur de la «patrimonalisation», de la «muséalisation», de la 
monumentalisation historique des traditions, toujours à conserver. Cette volonté systématique de monumentaliser atteste d'un véritable prurit notarial. L'art n'existerait en effet pour certains qu'embaumé, mis en conserve. Passé, dépassé, trépassé, il ne serait véritablement vivant que ressuscité dans des mausolées. Dès lors, il peut se visiter religieusement, touristiquement, comme on rend visite à nos morts dans le rituel silencieux d'un cimetière. Ainsi, pourrait-on toujours protéger l'art véritablement artistique comme une sorte de trésor précieux, un secret bien gardé. Comme le constipé Harpagon garde sa cassette, comme le fidèle protège des profanations le sanctuaire intouchable d'un sacré inviolable.

5 La profanation sacrilège serait en effet de ne pas saluer bien bas cette vertu première. Sa vertu première est en effet d'être toujours imité, répété, comme une sorte d'antériorité parfaite d'une authenticité identitaire. L'originalité ne serait jamais qu'à l'origine, à quoi il faudrait toujours faire retour. Tout ce qui succède à cette origine première, fonctionnant comme un modèle pour supprimer l'Histoire, est considéré comme une sorte de dégradation sinistre et ne pourrait se sauver aujourd'hui qu'en réitérant le geste inaugural de fort bon augure d'une création originelle, originaire, originale.

6 La conservation rituelle a alors pour finalité de constituer cette originalité originaire comme une sorte de paradigme à imiter, pour armer un retour archaïque à ce qui a bien commencé et doit donc toujours commander. Comme une source, comme une racine des liens d'une communauté ainsi retrouvée, d'une identité sans cesse magnifiée sur un territoire ritualisé, frontalisé, reconnu selon une hiérarchie ordonnatrice et ordinatrice.

7 Cette sacralisation aujourd'hui idéologiquement dominante d'un art « authentiqué »comme disait Diderot-, par une sorte de passé auréolé et sûrement pas olé-olé, autorise indiscutablement le retour d'une forme religieuse de l'art. Comme un recueil d'un absolu fondateur, pour relier des individus qui se retrouvent ainsi entre eux, toujours pareils aux mêmes, dans une sorte de célébration esthético-théologique, qui fait du grand artiste un grand auteur, le Dieu créateur d'une œuvre unique, absolue, et qu'il ne s'agirait que de répéter, d'imiter. A l'auteur de la Grâce, que nous avons connu, s'est désormais associée la grâce de l'auteur... Et l'esthétique muséale de la Grande œuvre porte en fait subrepticement le deuil de la théologie et se propose comme substitut de la mort des Dieux. Le rassemblement des chefs-d'œuvre est bien ecclésial. Il célèbre des victoires antécédentes, il capitalise des opulences, il immobilise, il fige, il fixe des perfections pour en faire, certes, le bien de tous, connu et apprécié de tous, c'est-à-dire une forme de liturgie religieuse. «Leitourgia » signifie étymologiquement "service public », un service public qui fait chacun se reconnaître, s'identifier, se rappeler, intérioriser les normes magnifiées de sa communauté.

8 Sachez bien cependant que le terme positif de "communauté » a un pendant négatif qu'on appelle le ghetto. Dès lors, dans cette communauté, pour se rappeler, il existe des monuments. De "moneo», qui veut dire "avertir». Le monument a en effet une fonction d'avertissement et il est une sorte de munition pour prévenir, prémunir et ainsi servir d'instrument d'inquisition des purs et des durs contre les subversions, les dispersions, le simili, le faux, l'artificiel, le pseudo, qui qualifieraient une modernité aujourd'hui disqualifiée.

9 Cette religiosité artistique d'un art embaumé dans son prestige est d'autant plus efficace-et je suis là encore volontairement polémique-qu'elle est associée 
parallèlement à une autre procédure de conservation, de protection, de patrimonalisation d'une autre matrice intouchable, d'une autre source sacrée de la perfection : la nature.

Elle-même intouchable, puisque source pure et ressource dure d'un ordre qui, comme chacun sait, ne ment jamais. Monuments historiques et sites classés entretiennent en effet la mémoire d'un idéal réel vers quoi se ressourcer pour réaliser à nouveau l'idéal parfait et ainsi faire la noce, à nos bels âges, à nos belles images, entretenir la flamme vivante de la nostalgie.

11 Sans démagogie, on ne doit donc que saluer les combattants actuels d'un art qui s'efforcent de faire vivre le spectacle comme un art vivant sous cette chape de plomb qui les neutralise avec des figures tutélaires d'une perfection passée. Certes, par provocation, délibérément, j'ai radicalisé à l'excès cette conception que je crois pourtant dominante, et donc dangereuse, de l'art actuel. Car il y a indiscutablement - je mets toujours de l'eau dans mon vin - une positivité du patrimoine. Et la muséologie a indiscutablement opéré depuis une quinzaine d'années une véritable révolution dynamique vers un néo-musée, qui renverse les perspectives reliquaires, fétichisantes et fossilisées, pour exposer bien au contraire ce qui a été évacué par l'art embaumé, pour réintroduire ce qui a été expulsé, ce qui a été conçu comme provocateur, ce qui a été ignoré, pour permettre alors des confrontations révélatrices, des croisements inédits, des parcours transversaux, tout simplement pour ouvrir le futur du passé.

12 Mais notre objet, c'est d'ouvrir l'avenir du présent. Comment donc réinventer véritablement une mémoire pour l'avenir, de façon à avoir une nouvelle intelligence du passé pour rendre vivant le présent, pour manifester un avenir de l'actuel? Que voulons-nous dire quand nous parlons de scènes et spectacles vivants en FrancheComté ? Qu'y a-t-il de vivant dans un art, dès lors que celui-ci se passe en FrancheComté ? C'est pourquoi je voudrais davantage réfléchir à ce terme de vivant.

13 Le vivant est donc un terme biologique, et non pas artistique. Le vivant, c'est un mélange de vital et de vivace. Le vital, c'est ce qui donne vie, le vivace redonne vie, redouble la vie qui par là même est vivifiée. Pour promouvoir quoi ? Pour promouvoir une vie de bonne santé, une vie bonne à vivre, qui augmente nos possibles, qui nous autorise à tenter le diable, à desserrer l'étau des contraintes, à ne pas nous enfermer dans la répétition monotone de la même vie. C'est ouvrir les alternatives possibles.

14 Ces trois dimensions de toute vie humaine que constituent selon Aristote le Survivre, le Bien-vivre et le Con-vivre, définissent aussi pour ce philosophe la vie pleinement humaine d'une humanité, qui alors est dite pleinement politique. Donc, l'art vivant n'est vivant que parce qu'il est d'abord inscrit dans une volonté politique.

15 En quoi consistent les trois dimensions du vivre, dans cette catégorie du politique qui définit proprement la philosophie d'Aristote ? Il s'agit alors de vivre ensemble selon ces trois dimensions dans un espace public qui permet l'exposition des possibles. De façon à pouvoir se développer selon des liens multiples, pluriels, en liaison avec un tout dynamique, lui-même relié selon Aristote à un cosmos, c'est-à-dire un ordre harmonieux d'une nature. Or, ce que nous apprend l'histoire de la philosophie politique, c'est que cette vitalité politique qui rend la vie vivable pleinement, qui permet à un homme d'être pleinement homme, d'être comme disaient les Romains, "viril ", n'est jamais donnée, inscrite dans un ordre préétabli, constituée par un modèle à imiter. Cette vitalité promotionnelle est acquise, ce qui veut dire aussi qu'elle peut donc se perdre. Elle se perd comment? En se dévitalisant. Elle se détruit 
comment? En se sclérosant dans l'imitation des modèles, c'est-à-dire en s'enfermant dans les mauvaises habitudes ou les tristes manies. Pour tout dire, en se normalisant dans le repli identitaire.

Ce dynamisme de la vitalité, en termes philosophiques, Nietzsche et plus tard Georges Canguilhem et François Dagognet l'ont appelé la normativité. Qu'est-ce que la normativité selon Nietzsche? C'est la vitalité créatrice de nouvelles normes. Plus simplement, c'est la vigueur, la vaillance de la création de ces valeurs. En quoi est-ce valeureux que d'être vivant? Le valeureux est celui qui est capable d'affronter les inédits, de rompre la monotonie de l'existence, de façon à affronter des infidélités à son propre milieu en rencontrant des milieux étranges, voire étrangers. Par là même qui est capable d'assumer le risque des rencontres inédites, de dépasser sans dommages ses propres limites stabilisées, de mettre en cause ses propres identités et, pour tout dire, de problématiser ses frontières. Jusqu'à prendre le risque de tomber malade, de se mettre en cause pour pouvoir se mettre en œuvre, c'est-à-dire se mettre en mouvement, ce qui est la définition de l'émotion.

L'émotion, c'est se mettre en mouvement, c'est mettre en mouvement son propre milieu de façon à y déposer sa marque, y actualiser ses puissances en préférant telle ou telle association, telle ou telle liaison ou telle ou telle relation, en élisant, sélectionnant, en choisissant, en excluant d'autres. La vie pleinement humaine, politique, désigne cette capacité de créer des nouvelles normes de façon artistique. En effet, et Nietzsche nous l'a montré, la création artistique est évidemment la dimension fondamentale de cette normativité valeureuse, vaillante, vigoureuse.

Une autre de ces dimensions - et là, je suis davantage expert -, c'est le sport.

Loin donc de la pieuse lamentation actuelle, on peut définir positivement l'œuvre d'art vivante et l'artiste vivant. L'artiste actuel n'est pas seulement négativement, comme nous l'impose de le penser une certaine philosophie et idéologie, celui qui aurait détruit les significations et qui aurait perdu le sens liturgique du sacré, incapable de nous révéler les essences d'un monde transcendant et de nous plonger dans les drames intérieurs d'une âme à la recherche de l'être authentique, du fond des choses, de l'idéal premier saisi par de grands antécédents. Aujourd'hui, et nous suivons ici F. Dagognet, l'artiste est celui qui positivement nous découvre un accès nouveau au réel, qui expérimente un insolite contact, qui explore un ingrédient inconnu pour libérer et offrir un substrat ignoré, riche de métamorphoses inconnues.

En dehors donc des discours amphigouriques de certains gourous, on peut faire une lecture positive et gratifiante des arts vivants contemporains qui, à partir des matières, des situations, des images, des scènes, ouvrent l'éventail infini des compositions, explorent le jeu des compossibles.

Cette analyse ne m'est pas personnelle et on trouve une illustration de cette positivité dans les analyses d'un Franc-Comtois célèbre mais tout à fait méconnu - Proudhon -, à propos de Gustave Courbet. Dans Principes de l'art, qui est une œuvre posthume jamais rééditée et donc jamais lue et encore moins relue, qui date de 1865, Proudhon dénonce l'exaltation d'un monde absolu, sanctuarisé, transcendant, séparé dans la pieuse célébration d'un bon souvenir, d'un monde grandiloquent, surchargé, évanescent. Il défend au contraire un art qui découvre le réel, qui le déplie, l'intensifie en le provoquant. Comment? En le concentrant, c'est-à-dire en l'inscrivant dans des séries de relations pour saisir des rapports, combiner des traits, associer des contraires, 
mélanger les identités, décloisonner les liens. Créer, pour Proudhon, c'est sérier, et vous savez qu'il est un grand philosophe de la série.

On pourrait donc s'inspirer de cette analyse franc-comtoise pour penser l'art vivant. Dans ces conditions, que voulons-nous dire à nouveau avec Proudhon, quand nous parlons de scènes et spectacles vivants en Franche-Comté ? Comment promouvoir l'art vivant sans le fétichiser, sans le régionaliser? En quels lieux normatifs, plastiques, multidimensionnels? Ce n'est pas un problème artistique, mais politique. C'est le rôle du politique que de produire des lieux vivants normatifs, où la pluralité des idées et des pratiques puisse s'exposer, se rencontrer et se confronter dans ses directions divergentes. Sans aucun sens obligé, sans aucun sens interdit, mais au contraire en multipliant les routes, les rencontres, les échanges, les paroles, les transports, les conjugaisons. C'est une fonction politique de rendre possibles ces confrontations dynamiques de la normalité. Tel est bien l'office du politique, c'est-à-dire le service que doit rendre le responsable politique, que seul peut le politique, sans asservissement ni servilité. C'est bien de cela qu'il doit répondre publiquement. Son autorité légitime est bien de fournir, de proposer les moyens et les lieux de cette augmentation publique, en autorisant l'exposition publique d'espaces pluriels, multiples, divergents.

Faust de Goethe au Volksteater, Berlin, 1982

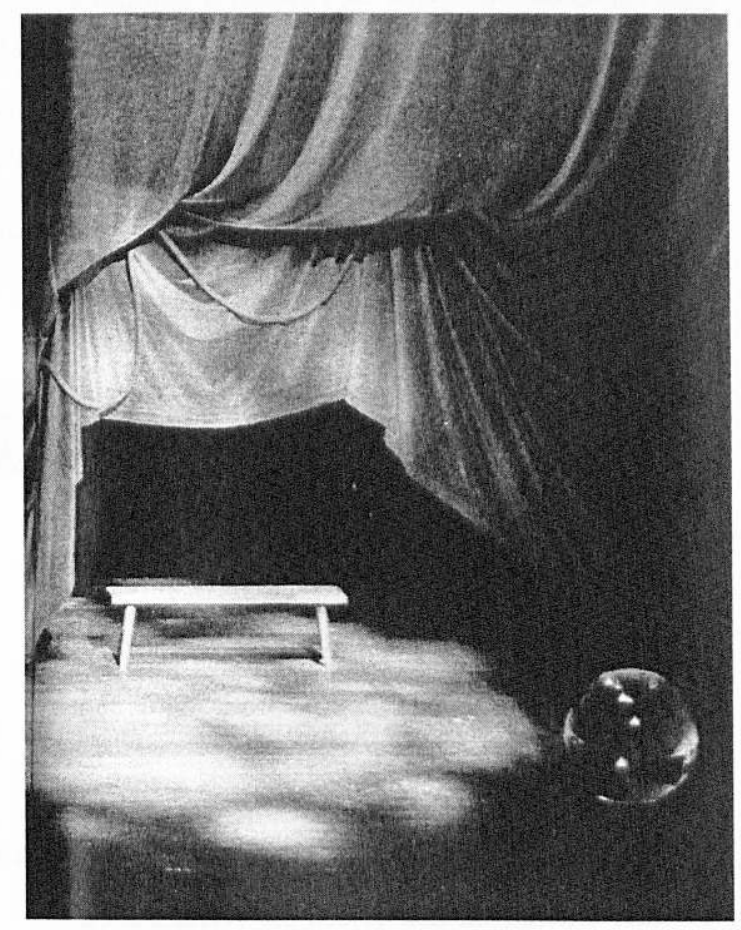

Photo Ruth Valz

23 Quels sont les critères qui peuvent alors organiser cette politique et par là même la donner à juger à nous, citoyens? J'en proposerai trois en analysant un exemple parmi d'autres de lieux publics où peut se jouer ce spectacle vivant d'une normativité politique: le gymnase dans la ville. Je le ferai en m'inspirant d'un philosophe et romancier américain, Richard Sennett, qui a beaucoup écrit sur la socialité politique de l'art vivant. Aussi bien dans des ouvrages consacrés à la ville, comme La ville à vue d'œeil, 
que dans des romans comme La soirée Brahms ou Port-Royal. Or, que nous dit Richard Sennett, qui est un élève de l'école de Chicago et qui est professeur à l'Université de New-York ? Le gymnase, tel que la philosophie grecque l'a pensé, a toujours eu un triple rôle d'exposition publique des divergences, des contradictions.

Le gymnase, c'est d'abord l'espace de la nudité corporelle, c'est un espace public où la beauté éduque le désir. Si peu enseignées, les dimensions morales du désir sexuel sont exposées aux yeux de tous. C'est aussi un lieu athlétique où le combat des forces, qui dégénère souvent en guerre, doit s'euphémiser en compétition selon des règles qui confèrent alors à notre liberté la puissance de s'augmenter en se mesurant à la puissance d'un alter-ego.

Et c'est enfin, troisième dimension, un espace de dialogue entre citoyens, où la confrontation des opinions peut se transformer en débats d'idées, mais là encore, selon une raison qui contrôle ces affirmations devant autrui en les publiant comme des arguments échangés, donc partageables par un interlocuteur. D'après Richard Sennett, cela signifie que dans un même lieu public, sinon peut-être dans un même geste, les Grecs-par exemple-ont inventé trois choses simultanément: la politique, la philosophie et l'athlétisme. Le public, le civil et le civique émergent donc selon des usages multiples que permet l'espace métamorphique du gymnase. J'aurais pu analyser d'autres lieux publics comme le stade, la scène de théâtre, le marché, le temple, la rue, la place publique, c'est-à-dire tout ce qui, à l'intérieur d'un espace public, définit le lieu de la citoyenneté. L'athlète qui s'entrainait, le joggeur aujourd'hui, est en fait un citadin qui s'expose au regard des autres. Et le citadin peut devenir, selon les hasards exigeants des rencontres, un citoyen qui discute de ses raisons de juger et de décider : c'est être souverain, donc être libre. Le même citoyen peut devenir quotidiennement plusieurs en un même lieu dont l'usage par chacun peut transformer la fonction. Trois dimensions, me semble-t-il, définissent l'effectivité pratique d'un lieu vivant politique et normatif.

Premier critère : un lieu vivant est véritablement politique, c'est-à-dire normatif, dans la mesure même où il permet la rencontre des inconnus. Car nous sommes submergés, catalogués par nos identités. Et la vertu d'un lieu public est de nous imposer le port du masque. De façon à être civil. Rien ne nous oblige à dire qui nous sommes, mais nous oblige à devenir quelqu'un d'autre que nous. La civilité préserve l'autre du poids du moi, elle consiste à traiter les autres comme des inconnus, à forger avec eux des liens sociaux respectant cette distance première. La civilité, c'est que des inconnus que nous rencontrons nous apportent un supplément, nous permettent de devenir autre que nous-même, indépendamment de nos qualités. Nous sommes des "hommes de condition », et pas des « hommes de qualité ».

Deuxième critère : la pluralité des usages. Un lieu n'est véritablement vivant que s'il permet la transformation de la fonctionnalité rigide des lieux, de manière à libérer une inventivité commune constitutive de liens que nous appellerons civiques, de façon à générer des relations fructueuses. C'est alors que, dans ces lieux, émerge la possibilité d'une histoire à raconter. Il s'y passe quelque chose dont on va pouvoir faire récit. Et cette transformation d'un lieu fonctionnel en un lieu autre peut être illustrée aussi bien par les taggers que par les rollers, qui occupent de manière constante des lieux qui ne sont pas faits pour cela. 
29 Troisième dimension : la palpitation des possibles. L'art politique, l'art vivant, est un art de la sympathie par lequel l'échange civil, civique, devient une relation proprement politique, constitue une identité partagée ensemble de façon à former momentanément, transitoirement - mais avec quels souvenirs ! -, un véritable " nous » collectif. Alors, nous occupons véritablement les scènes grâce à des spectacles par lesquels nous nous exposons en public.

30 Ces trois dimensions, ces termes - art, création, expression, exposition - tentent donc de définir la matrice d'une normativité publique, par laquelle une humanité se rencontrant, s'exposant, se liant à travers l'événement d'un récit, conquiert ainsi de manière incertaine, équivoque, éphémère sa propre normativité.

31 Utopie, me direz-vous? Certes. Tout cela relève bien de l'utopie. Et ces réquisits exigeants d'une politique ne peuvent, me dira-t-on, concerner une Région, car même l'Etat, aujourd'hui, ne parvient plus, ne parvient pas à l'instituer comme il le devrait. Certes, mais c'est justement en quoi cette utopie est proprement franc-comtoise... En effet, la Franche-Comté est d'abord et avant tout, dans l'histoire comme dans le Futur, une terre d'utopie. Sa vitalité, sa vivacité, son caractère vivifiant, ne sera-t-il pas de retrouver cet humus qui est sa véritable identité? Non pas celle figée d'un conservatoire sépulcral, mais celle dynamique d'une inventivité créatrice qui a fait toute sa vie, qui a fait toute la vitalité de son histoire, qui est bien sa source et sa ressource. Et qui définit donc bien l'axe de sa normativité. C'est en tout cas ce par quoi elle fut et demeurera vivante. Je vous remercie.

\section{AUTEUR}

\section{ROBERT DAMIEN}

Professeur de philosophie à la Faculté des lettres de Besançon, directeur du groupe de rechercheCNRS 1197 